\title{
Digitale Passagen und ihre Widersprüche. Ein Fazit
}

Die vorliegende Studie setzt sich mit medialen Phänomenen auseinander, die sich aus Praktiken der Digitalisierung von Filmen aus einer vordigitalen Produktionszeit ergeben. Gemeint sind Digitalisierungsphänomene von Filmen, die unter anderem als Archivfilme oder analoge beziehungsweise von mir als historische Filme bezeichnet werden; historisch deshalb, weil sie dem Zusammenhang einer früheren, zumeist vordigitalen Mediengeschichte entstammen. Es geht um Filme, denen ein Alters- oder Erinnerungswert zugeschrieben wird. Ansatz der vorliegenden Studie ist es, unter diesen Vorzeichen die Folgen und Konsequenzen für das Konstrukt ,Filmgeschichte im Sinne von medialen Geschichtsbildmodellierungen aus medien- und filmwissenschaftlicher Sicht beschreibbar zu machen. Die entwickelten Formen medialer Geschichtsbildmodellierungen verstehe ich in mehrfacher Hinsicht als Produkt und Faktor einer medienspezifischen Erinnerungskultur von und mit Filmen.

Besondere Herausforderung ist hier zuvorderst, Phänomene der Aktualisierung und der Wiederaufnahme - in meiner an Niney (2012) angelehnten Terminologie: Reprisen - von Filmen in der digitalen Domäne in ihrer dynamischen und nachdrücklich zeitgebundenen Dimension zu begreifen und analytisch fassbar zu machen: Digitalisate von Filmen und deren Editionen unterliegen den zumeist von Marktinteressen geprägten und daher auch bewusst sehr kurzen Entwicklungszyklen digitaler Technologien, welche ständige Migrationen und Updates er- und einfordern. Zudem treten die digitalisierten Filme auf der Distributionsebene aufgrund der sich ausdifferenzierenden, ihrerseits sich wandelnden Kanäle (DVD, Blu-Ray-Disc, Streaming, VoD) und Rezeptionsformen (Kino, Heimkino, Fernsehen, mobile Endgeräte) in eine sehr dynamische Zirkulation ein.

Meine Studie versteht sich vor diesem Hintergrund als eine medienarchäologische Untersuchung und zugleich als methodologische Reflexion der historischen Transitionsphase der letzten 25 Jahre, in der Praktiken und Anwendungen im Zusammenhang mit digitalen Technologien Einzug in die Filmrestaurierung, -archivierung, -distribution sowie -rezeption hielten. Aufgrund fehlender Standards und mangelnder Erfahrungen wurden in dieser Periode zwar zahlreiche ungelöste Fragen öffentlich diskutiert und vor allem auch implizit in den sich manifestierenden Phänomenen von digitalen Wiederaufnahmen von, älteren` Filmen ausgehandelt. Viele (kultur)politische 
Implikationen und Konsequenzen wurden und werden aber erst nach und nach deutlich. Dies gilt insbesondere für das Verhältnis analog/digital, das ich weniger im Zeichen oppositioneller Begriffe und Verfahren sehe als vielmehr im Zusammenhang mit differenztheoretisch begründeten medialen Verhältnissen. Ausgangspunkt ist das grundsätzliche Problem, wie sich die Sichtbarkeit und Erfahrbarkeit der suggerierten digitalen Qualität von digitalisierten Filmen sinnlich vermitteln.

Vor diesem Hintergrund hebt sich die vorliegende Studie vor allem methodisch von Untersuchungen ab, die ähnliche Gegenstandsbereiche berühren; hier sind etwa Studien zu nennen zur Digitalisierung von Filmen respektive zu Praktiken der Filmarchivierung und -restaurierung (u. a. grundsätzlicher Rodowick 2007, konkreter Fossati 2009); Analysen derVeränderungen der Filmrezeption insbesondere durch den Heimvideo- und Heimkinobereich (etwa Klinger 2006a; Distelmeyer 2012); Arbeiten zu den Konsequenzen in jüngerer digitaler Filmkultur (etwa Krautkrämer 2017; Hagener/Hediger/Strohmaier 2016; Einwächter 2014; Balcerzak/Sperb 2012 und 2009); nicht zuletzt Publikationen zu Fragen der Medien- und Filmgeschichtsschreibung (u. a. Elsaesser 2004 und 2002; Kessler 2011, 2006, 2002a und b, 2011; Schröter 2004a; Winkler 2000 und 1997) und zur mediatisierten Erinnerungskultur (Dijk 2007; Landsberg 2004/1996; Burgoyne 1999; Elsaesser 1996b).

Die vorliegende Studie greift gezielt spezifische Positionen und Erkenntnisse der angesprochenen Diskurse auf und entwickelt darüber anhand eigens recherchierten, ausgewählten und kompilierten Bildmaterials ihren analytischen Ansatz. Dieser ist dem Gegenstand entsprechend bewusst netzwerkförmig angelegt, stratisch-mehrdimensional ausgerichtet und clusterhaft organisiert, um letztendlich eine wahrnehmungstheoretische und -analytische Untersuchung durchzuführen, in der Fragen nach Erwartungshaltungen und Erfahrungsmodi konkreter Phänomene im Mittelpunkt stehen.

Aus zeit- beziehungsweise geschichtsphilosophischer Perspektive stellen mein Grundgedanke und auch die Begriffe Erwartung und Erfahrung eine medienwissenschaftliche Entlehnung von bestimmten Elementen aus Reinhart Kosellecks Überlegungen zur Semantik geschichtlicher Zeiten (1989) dar. Meine Untersuchung folgt im Argumentationsverlauf im Grundsatz einer ähnlichen Fragestellung wie Kosellecks Studie Vergangene Zukunft (1989: 11): So frage ich durchgängig danach, wie in einer bestimmten medialen Gegenwart zeitliche Dimensionen der Vergangenheit konstruiert und aufeinander bezogen werden. Die Hypothese ist dabei, dass sich in der Differenzbestimmung zwischen Vergangenheit und Zukunft - aus wirkungsästhetischer Perspektive: zwischen Erfahrung und Erwartung - eine Art ,geschichtlicher Zeit' fassen lässt. 
Die beiden Begriffe Erwartung und Erfahrung werden, so mein besonderer Ansatz, in einem phänomenologischen Horizont verortet; dies dahingehend, dass zwischen wahrnehmendem Subjekt und medialem Objekt ein gegenseitiges, sich prozessual entfaltendes Austauschverhältnis angenommen wird; ein in der Zeit dynamisches Verhältnis des gegenseitigen und gleichzeitigen Ergreifens wie Ergriffenwerdens.

Eine solche der Filmphänomenologie entlehnte Perspektive hat in den oben skizzierten Forschungsbereichen bisher kaum eine Rolle gespielt. Für den vorliegenden Erkenntniszusammenhang sollen dabei die Dimensionen der Verzeitlichung von Wahrnehmung, die meinen Befunden nach angesichts der Dynamik von Phänomenen und Entwicklungen als Denkmodelle unabdingbar sind, fassbar gemacht werden. Dieser Ansatz ist zugleich offen für jüngere leibphänomenologische Fragestellungen mit Blick auf das nutzende Subjekt.

Dies ist auch zu sehen vor dem Hintergrund eines grundlegenden, bereits oben angedeuteten Abbildungs- und Wahrnehmungsproblems, das sich im Kontext der Digitalisierung von ehemals analogen Filmen ergibt: Insbesondere bei der Digitalisierung von Filmen - so die ausdrückliche These dieser Studie bleibt die ,digitale Qualität an sich' oft implizit und vor allem nicht unmittelbar sichtbar oder kognitiv fassbar - jedenfalls nicht ohne die Inszenierung eines differenziellen medialen ,Vorher' -, weshalb die Transition auf wirkungstheoretischer Ebene als mediales Vermittlungsproblem begriffen und untersucht wird. Es wird angenommen, dass hier implizit eine mediengeschichtliche Entwicklung konstruiert und funktionalisiert wird - als Erwartungs- und Erfahrungshorizont. Im wahrsten Sinne des Wortes finden hier über filmische Erlebnisformen zeitlicher Differenzen Geschichtsbildmodellierungen statt.

In der Auseinandersetzung mit medialen Geschichtsbildmodellierungen im Horizont der Digitalisierung von Filmen ergibt sich eine weitere Besonderheit, die aus der Eigenschaft des Films entspringt, ein industriell (re)produzierbares und distribuierbares Medium zu sein, das zudem noch nachhaltig in Netzwerke einer kommerziellen Unterhaltungsindustrie eingebunden ist. Somit ist die Digitalisierung von Filmen nicht losgelöst von kulturindustriellen Interessen und ökonomischen Zwängen zu sehen. Dies beginnt mit der umstandslosen Nobilitierung und Aufwertung von digitalisierten Filmen unter dem nicht standardisierten, diffusen Güteetikett remastered.

Digitale Qualität erfüllt somit als kulturelle Manifestation eines Technoimaginären (H. Böhme 200o) die Funktion eines warenästhetischen Labels, das seinerseits zum Signifikanten einer teleologischen Fortschrittsideologie wird. Digitalisierungsprozesse, digitale Distributionsstrukturen, daraus entstehende Zugriffsformen und kommerzielle Interessen einer Unterhaltungsbranche werden vor diesem Hintergrund mit dem tradierten, für den vorliegenden 
Zusammenhang indes zu aktualisierenden Begriff Kulturindustrie aus der politischen Ökonomie zusammengebracht. Ich richte hierbei den Fokus vornehmlich auf den Zusammenhang von Kultur und Medien; mir geht es dabei weniger um den Nachweis eines totalitären Manipulationsmechanismus. Vielmehr interessiert hier die Modellierung der ästhetischen Dimension von Film als Ware, wobei auf grundsätzliche Überlegungen in Wolfgang Fritz Haugs Kritik der Warenästhetik (2009/1971) rekurriert wird. Haugs Analyse richtet den Fokus auf sinnliche Erscheinungen von Waren in ihrer ökonomisch spezifischen Form- und Funktionsbestimmtheit. Damit wird das ästhetisch konstruierte und vermittelte Gebrauchswertversprechen einer Ware, das der Tauschwertrealisierung vorausgeht, Gegenstand der Untersuchung (Haug 2009: 22 f.). In einem weiteren Schritt (hier insbesondere in $\rightarrow$ Kap. 6) wird dies mit Eva Illouz, einer Interpretin Haugs, um sinnlich-affektive Aspekte im Sinne eines ,Erfahrungskonsums' erweitert. Der Begriff wird in meiner Gedankenführung aus medienwissenschaftlicher Perspektive wirkungsästhetisch gewendet.

Die Studie verfolgt insofern das Ziel, den Prozess der Medientransition nicht lediglich als eine technologische von analogem zu digitalem Film anzugehen, sondern vielmehr dessen vielschichtige, medienspezifisch erinnerungskulturelle Dimension - verstanden als filmisch-medialer Erlebnishorizont - beschreibbar zu machen. Dies grundiert die grundsätzlichen - in den $\rightarrow$ Kap. $2-7$ diskutierten - Ausgangsfragen:

- Wie lässt sich digitale Qualität von (Bewegt-)Bildern überhaupt fassen?

- Über welche Formen und unter welchen Bedingungen wird digitale Qualität eines historischen Films wahrnehmbar (gemacht)? Wie vermittelt sich in Differenz dazu eben auch eine Dimension der Geschichtlichkeit des wiederaufgenommenen Films?

- Welche Rolle spielt hierbei die Vermittlung eines differenziellen Vorher und Nachher beziehungsweise des Gegensatzes von alt und neu, der oft auch mit analog/digital identifiziert wird? Gegenstand sind insofern konkrete Phänomene der digitalen Wiederaufnahmen, an die nicht allein die Frage gestellt wird, was an ihnen neu ist, sondern darüber hinaus, wie sie uns unter bestimmten Bedingungen vermitteln, dass sie neu seien.

- Welche Funktion übernimmt jeweils die mediale Konfiguration und Vermittlung des Differenzverhältnisses?

Und daran anschließend:

- Welche Konsequenzen zeitigt dies in der wirkungsästhetischen Dimension für die Erfahrung zeitlicher Verhältnisse - nicht nur auf einer medienhistoriografisch lesbaren und wirksamen Ebene, sondern generell auch mit Blick auf ein medial modelliertes Bild von Geschichte? 
Hervorzuheben ist: Bei der Beschäftigung mit diesen Fragen bediene ich mich eines medien-, vor allem filmwissenschaftlichen Instrumentariums, wobei Verfahrensweisen und Positionen der Film- und Bewegtbildanalyse verbunden und auf Dispositive und Aufführungsformen von Filmen heute ausgeweitet werden. Die Analysen von Digitalisaten (= digitalisierten Filmen), digitalen Editionen und digitalen Dispositiven von Filmen nehmen insofern eine verflochtene, vernetzende Perspektive auf die involvierten medienästhetischen und wahrnehmungskonfigurierenden Dimensionen ein. Der Ansatz profiliert sich konkret über die analytische Berücksichtigung der Kombination von drei Schichten, die im Modus der digitalen Wiederaufnahme die Erfahrung von medialer und filmischer Zeitlichkeit beziehungsweise zeitlicher Differenz prägen. So werden die folgenden Ebenen aufeinander bezogen:

a. die konkrete, unmittelbare Bewegtbildwahrnehmung; b. die medial umgebenden Konfigurationen des jeweiligen Dispositivs als Bedingung der Erscheinung der Bilder; c. die überformenden und rahmenden Diskurse insbesondere im Kontext ihrer technikgeschichtlichen sowie ökonomischen Pragmatik.

Grundsätzlich lassen sich meine Darstellungen in den Analysekapiteln $\rightarrow$ Kap. 4-7 als Auslegungen von medienhistoriografisch wirksamen Aspekten begreifen, die zusammen ein gemeinsames pragmatisches und phänomenologisches Wahrnehmungsmodell angesichts von digitalen Reprisen von Filmen entwerfen. In $\rightarrow$ Kap. 4, 5, 6 und 7 wird durch die Unterschiede in den Gruppen ästhetischer Analyseobjekte, welche jeweils andere Aspekte fokussieren und sichtbar machen, die Aufmerksamkeit auf unterschiedliche funktionale Einordnungen und damit verschiedene Diskurskreise gelenkt. Hier wird deutlich, dass das Problemfeld digitale Reprisen nur in einer polyperspektivischen Herangehensweise in seinen zahlreichen Facetten und Implikationen fassbar gemacht werden kann.

Ich sehe die theoretische und kulturkritische Auseinandersetzung mit Phänomenen digitaler Reprisen bestimmt von einem Netz von Begriffen. Letztere stehen zunächst ahierarchisch nebeneinander und wollen situativ, je nach Kontext für die analytische Profilierung eines Aspekts, aufeinander bezogen werden. Obwohl die in Anschlag gebrachten Ansätze sich zum Teil unterschiedlichen Voraussetzungen verdanken, weisen sie eben doch auch wichtige Schnittmengen auf: Dazu zählen die Betonung performativer Wahrnehmungsund Sinnbildungsprozesse sowie die wiederkehrende Bedeutung der Wechselwirkungen zwischen Aktuell-Präsentischem und Virtuell-Imaginärem.

So zeitigen die $\rightarrow$ Kap. 4-7 grundsätzlich eine doppelsträngige Argumentationsstruktur, indem sie jeweils a. ausgewählte ästhetische Strategien anhand 
von Phänomenen digitaler Reprisen untersuchen und herauspräparieren und b. die entsprechenden, auch von kulturindustriellen Kontexten imprägnierten Erfahrungs- und Erwartungshorizonte in Hinblick auf ihre Formen von Geschichtsbildmodellierungen dimensionieren.

Vor diesem Hintergrund lassen sich die folgenden Befunde zur Wahrnehmungsmodellierung angesichts digitaler Reprisen von Filmen, denen Alters- und Erinnerungswerte zugeschrieben werden, zusammenfassen:

- Es drängt sich auf, digitale Wiederaufnahmen von Filmen insbesondere in ihrer Schichtung verschiedener zeitlicher Ebenen als eine spezifische Ausprägung von Zeitbildern im Deleuze'schen Sinne zu konzeptualisieren: In zeit- und filmbildtheoretischer Perspektive lassen sich Funktionsweisen von zeitlich wechselseitigen Bildern beobachten, die memophänomenale Effekte beim Zuschauer mit Erwartungshaltungen und Erfahrungsmodi generieren. Eine solche medienhistoriografische Erfahrungsbildung verbindet sich mit einer spezifischen wirkungs- und rezeptionsästhetischen Fetischisierung von Bewegtbildern. Besonders augenscheinlich wird dies in Filmformaten, die neue Bildtechnologien bewerben (vgl. die Analysen in $\rightarrow$ Kap. 4).

- Filme sind in ihrer situativen Erscheinungsform im Horizont digitaler Wiederaufnahmen als performativ-ereignishaft zu verstehen. Dies wird besonders deutlich bei den in $\rightarrow$ Kap. 5 besprochenen Beispielen, die in unterschiedlichen Variationen jeweils eine tatsächliche Aufführung von (frühen) Filmen thematisieren und problematisieren. Dies wiederum weist auf die Bedeutung des konkret körperlich miterlebenden Zuschauers, was für mich zu der Modellierung eines phänomenologischen, auf die leibliche Gegenwärtigkeit hin ausgelegten Wahrnehmungsmodells angesichts digitaler Wiederaufnahmen (hier auch als digitale Performance bezeichnet) führt. Mein Wahrnehmungsmodell ist einmal mehr gebunden an die Vorstellung performativ interagierender, differenzieller Zeitschichten, die Dimensionen des Gegenwärtigen und Vergangenen bestimmen. Ein kuratorisches Konzept wird hier von mir wirkungsästhetisch gewendet: Bridging the gap, marking the difference (Kessler 2011). In dieser Perspektive wird der Zuschauer als körperliche (Nach-)Vollzugsinstanz von medial vermittelten Eindrücken von Geschichtlichkeit konzipiert. Die historisierende Referenzierung eines Vorher und Nachher wird immer prozesshaft konstituiert und damit zugleich vom Wahrnehmenden im Somatisch-Sinnlichen - im Erlebnis - vollzogen. Dies bezeichne ich in Anschluss an G. Böhme als Formen einer aisthetischen Historiografie im Horizont ereignishafter Wiederaufnahmen von Filmen. G. Böhmes Konzept von Aisthetik (2001) erscheint mir in Verbindung mit Phänomenen der ästhetischen Wahrnehmung auch deshalb produktiv, weil es sich über den Kunstbereich hinaus auf 
lebensweltliche - zumal kulturindustrielle - Zusammenhänge beziehen lässt. Hier setzt meine Formulierung an: Erinnerungskulturelle Phänomene der digitalen Reprise historischer Filme werden in aisthetischer Perspektive gesehen und in ebendiesen aisthetischen Wirkungsformen als geschichtsbildmodellierend - über eine abstrakt medienhistoriografische Wirksamkeit hinaus - begriffen.

- Digitale Wiederaufnahmen von Filmen sind auf jeweils bildlich-manifeste Funktionalisierungen des Imaginären zu untersuchen. Denn: Jeder Film basiert - spätestens seit Christian Metz - auf dem Imaginären. Besonders gilt dies für illusionsbildende Spielfilme. Jede Wiederaufnahme von Filmen funktionalisiert Erfahrungen von Zeitdifferenz. Digitale Reprisen vermitteln Zeitdifferenzen konnotiert mit dem Technoimaginären des Digitalen. So gilt es, den Fokus vor allem auf Phänomene zu richten, wenn das Technoimaginäre der digitalen Reprise gekoppelt wird mit illusionsbildendem Potenzial von Filmen. So bereichert das Imaginäre, das seine Qualität als illusionsbildende Kraft im Spielfilm am stärksten zur Geltung bringt, Vorstellungen raumzeitlicher Ordnungen von Geschichte und Zukunft um das Hypothetische, Utopische, Spekulative, Phantastische, Virtuelle in besonderer Weise.

- Solche Geschichtsbildmodellierungen funktionalisieren das Imaginäre als Grundprinzip filmischer und bildlicher Darstellung und Wahrnehmung. Ich analysiere dies konkret anhand audiovisueller Paratexte in digitalen Editionen. Hier wird über die Anwendung semio-pragmatischer Ansätze die Legierung der Register von präexistenten, re-kontextualisierten Spielfilmbildern und Non-Fiction-Bildern deutlich. Über Beispiele, in denen Bildzitate aus phantastischen Spielfilmszenen nicht nur zur sinnlichen Vermittlung des Technoimaginären beitragen, sondern zudem auch noch als mediatisierte, mit utopischen Elementen aufgeladene prothetische Erinnerungen dieses erlebbar machen, werden Funktionsweisen der Inkorporierung filmischer Fiktionen anschaulich. Dabei ist die Verschränkung von individueller und kollektiver Erinnerung im Wahrnehmungsakt als besonders bedeutsam anzusehen.

- Digitale Wiederaufnahmen von Filmen in ihrem Status als filmische Kunstwerke sind von Erwartungshaltungen und Erfahrungsmodi der Relationalität geprägt. Konkret bedeutet dies, dass in dem ausgreifenden abschließenden $\rightarrow$ Kap. 7 die Bedingungen medialer Konstellationen der digitalen Reprise als - wie ich es nenne - Mise en Relation in den Blick genommen werden. In wirkungsästhetischer Perspektive werden Kontextualität und Relationalität als medienhistoriografisch erfahrungsbildend analysiert. Dabei wird von dem medienhistorischen Phänomen der Versionenhaftigkeit von Filmen ausgegangen, welche unter anderem auf der schon immer beim Film 
gegebenen Reproduzierbarkeit - ob nun ,technisch` oder digital - gründet. Diese Eigenschaft des Films verbindet sich nun mit Grundprinzipien der digitalen Domäne - insbesondere mit verräumlichten Netzwerkstrukturen. Vor diesem Hintergrund legt das $\rightarrow$ Kap. 7 anhand unterschiedlichster Beispiele dar, dass die Erwartungshaltung, aber auch das tatsächliche Erlebnis des Vergleichens eine entscheidende Rolle für die jeweilig vom Zuschauer/ Nutzer erlebte Geschichtsbildmodellierung einnimmt. Dabei besteht einmal mehr mein besonderer Ansatz darin, die Formierungen nicht allein auf der metafilmischen Ebene - etwa der Überlieferungsgeschichte von Filmen - zu sehen. Darüber hinaus werden auch die strukturellen und ästhetischen Eigenschaften der wiederaufgenommenen Filme - unter Einschluss der in ihren diegetischen Universen verhandelten Themen - als Erlebnishorizont(e) nicht außer Acht gelassen; im Gegenteil: Filmwerke als mehrschichtig zu verstehende Cluster sind integraler Bestandteil der von mir profilierten Aspekte medienhistoriografischer Erfahrungsbildung, das heißt mithin der jeweiligen Geschichtsbildmodellierungen.

Vor dem Hintergrund dieser Befunde aus $\rightarrow$ Kap. 4-7 sind noch zwei grundsätzlichere konzeptionelle Bemerkungen zu dem Verständnis des untersuchten Gegenstandes, zu der methodisch-begrifflichen und strukturellen Auseinandersetzung mit ihm von Bedeutung: Zunächst verstehe ich die jeweiligen Phänomene und Konfigurationen digitaler Wiederaufnahmen in Anlehnung an Benjamin (vgl. Zwischenfazit in $\rightarrow$ Kap. 3.9) als prozessuale Passagen. Bei Benjamin (1982) wird die Auseinandersetzung mit Erinnerung und Geschichtsschreibung als archäologische Tätigkeit beschrieben, die sowohl dem Modell einer zeitlichen Schichtung als auch dem Prinzip von Sichtund Erkennbarkeit im Fluss der Zeit verpflichtet ist. Dies übernehme ich, da, wie einleitend zu diesem $\rightarrow$ Kap. 8 konstatiert, geschichtsbildmodellierende mediale Konstellationen in einem ständigen Fluss gesehen werden müssen in dialektischer Verwebung mit den Tendenzen und Interessen der Gegenwart.

Um insofern der vielschichtigen Zeitlichkeit der Phänomene digitaler Reprisen Rechnung zu tragen, werden in den $\rightarrow$ Kap. 4-7 die Fallstudien von Wiederaufnahmen als clusterförmig strukturierte Komplexe untersucht. Die clusterförmige Betrachtung ist deshalb gewählt, weil es sich um beziehungssetzende Wahrnehmungskonfigurationen und Erlebensmodi handelt, die über kopräsente Schichtungen unterschiedlicher Zeitebenen funktionieren.

So entwickeln sich sowohl die Makro- als auch die Mikrostruktur innerhalb der Kapitel nach dem Prinzip der Auffächerung von möglichen Perspektiven und theoretischen Zugriffsmöglichkeiten. Angesichts der unterschiedlichen beteiligten Disziplinen und Bereiche von Digitalisierung und digitaler Distribution/Edition/Zirkulation von Archivfilmen lässt sich das Feld nicht linear 
darstellen und argumentativ entfalten. Vielmehr scheint eine Struktur angemessen, die emblematisch im Bild eines Kristalls gefasst werden kann: Je nach Standort variieren die Lichtbrechungen und bringen andere Reflexionslinien zur Anschauung. Dies wird im Argumentationsaufbau besonders deutlich, wenn Begriffe unterschiedlich akzentuiert und auf verschiedenen analytischen Ebenen diskutiert werden (vgl.v. a.Performance und Imagination, $\rightarrow$ Kap. 5 und $\rightarrow$ Kap. 6). Auch wird dies sichtbar in der netzartigen Verweisstruktur, in der immer wieder versucht wird, auf Querverbindungen der einzelnen Kapitel untereinander hinzuweisen.

Inhaltlich schließt die vorliegende Studie in ihrer Grundtendenz an den kulturkritischen Ansatz H. Böhmes (2012) mit seiner Analyse der Moderne im Zeichen des Fetischismus an. Moderne - in der vorliegenden Studie verstanden im Horizont gegenwärtiger medialer Praktiken und Phänomene - ist in ihrer Widersprüchlichkeit zu begreifen. Im Folgenden sollen exemplarisch einige dieser Widersprüchlichkeiten, die bei der Untersuchung von medienhistoriografisch wirksamer Erfahrungsbildung in Hinblick auf das Konstrukt Filmgeschichte zutage traten, zusammengefasst werden:

- Bei den untersuchten Funktionsweisen fällt auf, wie sehr ganz unterschiedliche Ausformungen und Variationen Wahrnehmungsmodi offerieren, die im Dienste des Technoimaginären fetischisierende und mystifizierende Elemente zeitigen. Dies steht in einer Linie mit Diagnosen H. Böhmes zur Kulturgeschichte der Technik und den sich dort artikulierenden eschatologischen Fortschrittshoffnungen - mit der spezifischen Verbindung von instrumenteller Rationalität und mythischer Beherrschung der Natur. Meines Erachtens ist dies im Zusammenhang mit einer Technikgeschichte, die sich filmbildlich vermittelt, wahrnehmungstheoretisch zu re-formulieren. Im vorliegenden Gegenstandsbereich werden Bildformen und Anordnungen beschworen, die digitale Versionen von Filmen als die Realität transzendierende Erfahrung präsentieren. Dies ist aufschlussreich, da damit, wie in jeweils unterschiedlichen Facetten im $\rightarrow$ Kap. 4 und $\rightarrow$ Kap. 7 erläutert, dem Glauben als sakralisierender Wahrnehmungshaltung seitens des Konsumenten/Nutzers/Rezipienten angesichts digitaler Bilder und Phänomene eine wesentliche Rolle zukommt - auch in der historisierenden und ästhetischen Wertzuschreibung an die Filmbilder. Mit dem Technoimaginären der digitalen Domäne verbindet sich im Zusammengehen mit Filmbildern einerseits eine auf Kommendes ausgerichtete Erwartung; andererseits ist es ein sich der filmischen Bewegtbildillusion und -wirkung verdankender medialer Wahrnehmungs- und Erlebnishorizont. In Anlehnung an H. Böhme formuliert: Die digitale Qualität eines Filmes und einer Zugriffsform ist nie das, was von sich aus immer schon digital ist, sondern es muss der Glaube 
als spezifischer Wahrnehmungsmodus erzeugt werden, dass die Qualität mehr ist als nur ein numerisches Prinzip der Datenverarbeitung. Erst eine solche Fetischisierung mit ihrem - bei jedem Fetischismus wirksamen „erregenden Zauber“ (H. Böhme 2012: 394) schafft jenen sinnlichen Erwartungshorizont, der sich dann mit dem Filmisch-Imaginären überlagert, das seit jeher von dem Doppelcharakter geprägt ist, sowohl Wahrnehmungs- als auch Vorstellungsbilder gleichzeitig dem Zuschauer zu offerieren.

Der hier bemühte Glaube ist, so mein Modell, auch mehr als eine Lektüreanweisung, denn Glaube als Wahrnehmungsmodus bezieht sich auf eine zum Teil tatsächliche körperliche Erfahrung der transzendental aufgeladenen Überschreitung. Insofern dienen hier Überlegungen aus der Filmphänomenologie dazu, die präsentisch-leibliche Sinndimension in ein Wirkungsmodell von Geschichtsbildmodellierungen im Zeichen einer technikgeschichtlichen Transition zu integrieren. Dies ist umso pikanter, als es sich um eine Medientechnologie handelt, die schon früh im Zeichen eines „Mythos der Interaktivität" stand (Manovich 2001).

- Darüber hinaus - dies steht in engem Zusammenhang mit dem vorherigen Punkt - zeichnen sich in allen Kapiteln immer wieder Übertragungsmechanismen aus der bewegtbildlich vermittelten Fiktion auf die metafilmische Überlieferungsgeschichte sowie die aktuellen Erscheinungs- und Nutzungsformen ab, die zusammen ein Cluster ausbilden. Über die gegenwärtigen Gebrauchs- und Zugriffspraktiken erfahren diese Cluster, so die Befunde, zum einen zunächst eine diskursive Ausdehnung auf lebensweltliche Bereiche (oder gar Anthropomorphisierung wie im Beispiel Weltenbrand: Film als Soldat an der Geschichtsfront). Darüber hinaus werden aber auch über verschiedene wirkungsästhetische Strategien, über die Vermittlung prothetischer Erinnerungen Geschichtsbildmodellierungen vorgenommen. Vor allem in $\rightarrow$ Kap. 6 wurde die Verbindung von Méliès' Le Voyage DaNS LA LUNE mit der tatsächlichen Mondlandung beschrieben, die über ganz unterschiedliche Bildregister vermittelt ist. Variiert findet sich dieses Prinzip in $\rightarrow$ Kap. 7 diskutiert, wenn über die DVD-Edition von DiE ScHöNSTE und deren Schauwerte ex negativo auf historische Zensurpraktiken und den Wandel im politischen Klima geschlossen werden soll/kann.

Bemerkenswert ist in diesem Zusammenhang (insbesondere etwa in den Fallstudien $\rightarrow$ Kap. 6), dass das in Bildern zum Teil spektakulär ausgestellte Filmisch-Imaginäre zur Geschichtsbildmodellierung verwendet wird. Kracauer (1984/1947) hat mit Blick auf Filme das Produktive vor allem auch darin gesehen, dass diese als Ausdruck tiefenpsychologischer Dispositionen und Bedürfnisse einer Gesellschaft gelesen werden könnten, da sie das Gesellschaftlich-Imaginäre vermitteln (Kracauer 1984: 7). Eine These meiner 
Studie lautet, dass sich dies wie folgt in einer Variation produktiv für die Disposition angesichts von Phänomenen der Digitalisierung des Filmerbes modifizieren lässt: Digitalisate und digitale Dispositive inkorporieren das filmisch Imaginäre als prothetische Erinnerung und vermitteln diese als Geschichte; ein Zusammenhang, der immer kritisch in seinem spezifischen kulturindustriellen und erfahrungskonsumistischen Kontext zu reflektieren ist.

- Dies führt zu einer widersprüchlichen Konfiguration, die sich bereits im Titel des zentralen und ausführlicheren $\rightarrow$ Kap. 7 wiederfindet: Wie ist mit der Spannung umzugehen, wenn bestimmte mediale Konfigurationen im Sinne einer Do-it-yourself-Suggestion zeitliche, (historisch) bedeutsame Veränderungen in den Nachvollzug eines Nutzers verlegen? Dies wurde besonders deutlich in Bildkonstellationen, die in $\rightarrow$ Kap. 7 als erlebte Mise en Relation angesichts von Split-Screen-Konstellationen thematisiert wurden, in denen historische Veränderungen über den Nachvollzug seitens des Nutzers mit dem Cursor auf der Bildebene herbeigeführt werden. Dies hat eine zeiträumliche Relativierung historischer Wahrnehmungsmodi und der damit verbundenen historiografisch wirksamen (leiblichen) Erfahrungsbildung des Subjekts zur Folge. So hat das Versprechen Do-it-yourself-History wahrnehmungstheoretische, darüber hinaus aber auch grundsätzlichere geschichtspolitische Konsequenzen, die stets im Blick behalten werden müssen.

Mein Fazit ist vor allem ein methodologisches: Die genannten Aspekte sind in jedem gegebenen Kontext in ihrer Funktionsweise und mit Blick auf die Konstitution eines historischen Subjekts kritisch zu hinterfragen - nicht zuletzt angesichts von extrem dynamischen und flüchtigen medialen Konfigurationen. Dabei gilt es, die zweifache Schichtung im Blick zu behalten: Die medialen Konfigurationen generieren sowohl Erwartungshorizonte, in denen sich Technoimaginationen digitaler Medien spiegeln, als auch darüber hinaus immer wieder unmittelbar wirksame Erlebnissphären.

Inwiefern eine solche mediale Vermittlung von zeitlicher Veränderung als Nachvollzug brisante politische Dimensionen in sich birgt, soll ein letztes Beispiel aus der jüngsten Zeit aufzeigen, in dem ein Bild in einer geschichtspolitischen Dimension zum Bewegtbild sowie - in meiner Lesart - zum den Zuschauer bewegenden Bild wird. Mit diesem Beispiel verlasse ich abschließend den Bereich der Filmtheorie und -geschichte und erweitere mein Instrumentarium punktuell auf lebensweltliche Zusammenhänge. Eine der in $\rightarrow$ Kap. 7 beschriebenen Bildkonfigurationen zur Foto- und Filmgeschichte ähnliche Konstellation wurde am 13.11.2016 auf der Webseite von Human Rights Watch veröffentlicht unter der Überschrift „Iraqi Kurdish Forces' Destruction 
of Villages, Homes in Conflict with ISIS“. Man kann hier auf der Webseite von Human Rights Watch die Satellitenbilder vom 14. Februar 2015 eines noch aus augenscheinlich bewohnten Häusern bestehenden kurdischen Dorfes durch eine wischende Cursorbewegung mit einem weiteren Satellitenbild ,überschreiben', das am 20. Mai 2015, also Monate später, aufgenommen wurde. In diesem sind keine Gebäude mehr zu sehen (Abb. 8.1).

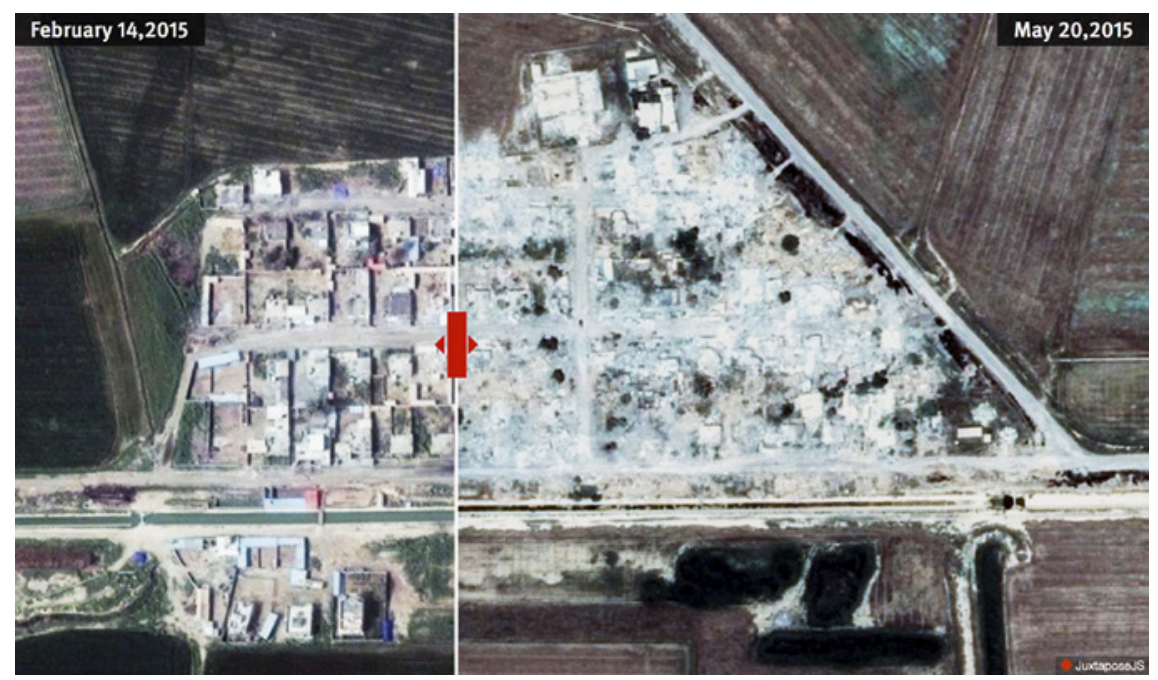

Abb. 8.1

Durch diese Veränderung des Bildes kann man vergleichend nachvollziehen so die diskursiv-paratextuelle Rahmung -, dass das Dorf in dem angegebenen Zeitraum zerstört worden ist. Für den nutzenden Leser vermittelt sich die Bedeutung über das Intervall, die zeitliche Leerstelle in der leiblich-physischen Wischbewegung zwischen einem bildlichen Vorher und einem Nachher. Dies zeitigt höchst brisante politische Implikationen, da über diese Bildkonfiguration die Zerstörung kurdischer Dörfer durch die irakische Armee dokumentiert werden soll. Die Referenz wird medial vom Nutzer gestiftet durch die Handbewegung von links nach rechts. Allerdings würde eine Bewegung nach links den Aufbau ebendieses zerstörten Dorfes nahelegen - eine andere Geschichte.

Was hier zum Ausdruck kommt, ist das implizit Politische der medialen Konfiguration und der Modellierung eines Nachvollzugs zeitlicher Differenz im Modus des Transitorischen angesichts der Veränderbarkeit von Bildern. Dies geht historisch zurück auf das Medium Film, das bereits - wenn auch unter etwas anderen Vorzeichen - aufgrund seiner bewegtbildlichen Qualität, 
der damit einhergehenden Erfahrung einer präsentischen Bewegung und der Möglichkeiten der Montage Kategorien von Zeit- und Geschichtlichkeit neu konfiguriert hat. Nun erfahren Bewegtbilder Wiederaufnahmen und zusätzliche Re-Konfigurationen im Zeichen ihrerseits modellierender Dispositive, die sich wiederum selbst nur als mediale Passagen entpuppen.

Vor diesem Hintergrund ergeben sich meine Schlussfolgerungen aus der Betrachtung gerade der letzten 25 Jahre, aus der Reflexion des Umgangs mit dem überlieferten Reservoir an (unterhaltungs)industriell hergestellten und zirkulierenden Bewegtbildern; einer Phase der technologischen Umwälzung, welche nachdrücklich auch von kulturellen Imaginationen und Selbstentwürfen befeuert wurde und immer noch wird. Grundlegend für mich ist: Das politisch-ideologisch Formierende der jeweiligen sicht- und wahrnehmbaren Phänomene von Film- und Bildgeschichte ist zu suchen

a. in der jeweiligen Konzeption unterschiedlicher (auch digital-technoimaginär geprägter) medialer Dispositive,

b. in den Praktiken der Mise en Relation von leiblicher Präsenz, zeitlicher Nähe und Distanz,

c. in der situativen Etablierung einer unpersönlichen, geschichtsevozierend wirksamen (Enunziations-)Instanz.

In diesem dreidimensionalen Gefüge sind die jeweiligen Konfigurationen stets auf ihre Modi der bewegtbildlichen Illusionsbildung hin kritisch zu überprüfen. Dies gilt ebenfalls für zukünftige mediale Entwicklungen und deren Formen der Wiederaufnahme von Filmgeschichte(n).

Erkenntnistheoretisch gesehen, geht es um die Bedeutung situativer, interessegeleiteter Konfigurationen von historischer Zeit. Diese unterliegen, um in digitalen Medienumgebungen sicht- und erfahrbar zu bleiben, immer einem kontinuierlichen differenziellen Wandel. Davon betroffen ist auch das Konzept eines sich als historisch, zeitlich verortet erlebenden Subjekts angesichts von Bewegtbildern und filmischen Formen.

All die genannten Parameter sind in ihrer zeitlichen Dynamik zu sehen eingebunden in das kulturindustrielle Prinzip ,Update!. 